\title{
Superficial venous thrombosis: disease progression and evolving treatment approaches
}

This article was published in the following Dove Press journal:

Vascular Health and Risk Management

30 August 2011

Number of times this article has been viewed

\section{Maria E Litzendorf \\ Bhagwan Satiani}

Heart and Vascular Center and Division of Vascular Surgery, Department of Surgery, The Ohio State University College of Medicine, Columbus, $\mathrm{OH}$, USA
Correspondence: Maria Litzendorf Division of Vascular Surgery, Department of Surgery, The Ohio State University Medical Center, St 3018, 456 West, 10th Avenue, Columbus, OH 43210, USA

$\mathrm{Tel}+\mathrm{I} 6142938536$

Fax + I 6142938902

Email maria.litzendorf@osumc.edu

\begin{abstract}
Treatment of superficial venous thrombosis (SVT) has recently shifted as increasing evidence suggests a higher than initially recognized rate of recurrence as well as concomitant deep venous thrombosis. Traditional therapies aimed at symptom control and disruption of the saphenofemoral junction are being called into question. The incidence of deep venous thrombosis has been reported to be $6 \%-40 \%$, with symptomatic pulmonary embolism occurring in $2 \%-13 \%$ of patients. Asymptomatic pulmonary embolism is said to occur in up to one third of patients with SVT based on lung scans. The role of anticoagulation, including newer agents, is being elucidated, and surgical disruption of the saphenofemoral junction, while still an option for specific cases, is less frequently used as first-line treatment. The individual risk factors, including history of prior episodes of SVT, the presence of varicosities, and provoking factors including malignancy and hypercoagulable disorders, must all be considered to individualize the treatment plan. Given the potential morbidity of untreated SVT, prompt recognition and understanding of the pathophysiology and sequelae are paramount for clinicians treating patients with this disease. A personalized treatment plan must be devised for individual patients because the natural history varies by risk factor, presence or absence of DVT, and extent of involvement.
\end{abstract}

Keywords: superficial venous thrombosis, progression, treatment

\section{Introduction}

Superficial venous thrombosis (SVT) has received increased attention as more clinicians are recognizing the potential morbidity of untreated disease. Traditional therapies aimed at symptom control and disruption of the saphenofemoral junction (SFJ) are being called into question. The incidence of deep venous thrombosis (DVT) has been reported to be $6 \%-40 \%$, with symptomatic pulmonary embolism occurring in $2 \%-13 \%$ of patients, and asymptomatic pulmonary embolism occurring in up to one third of patients with SVT based on lung scans. ${ }^{1}$ Given the potential morbidity of untreated SVT, prompt recognition and understanding of the pathophysiology and sequelae are paramount for clinicians treating patients with this disease. In addition, a review of current strategies involving newer and developing treatment approaches is warranted.

\section{Epidemiology and pathophysiology}

SVT has been reported to occur in approximately 125,000 people yearly in the US. ${ }^{2}$ However, this is generally believed to underestimate the true incidence, because many cases are unrecognized and unreported. Some studies demonstrate a higher prevalence in women overall, as well as an increased incidence with age in both males and females. ${ }^{3,4}$ Varicose veins, the most frequent predisposing factor, are present in 
up to $62 \%$ of patients with SVT. ${ }^{5,6}$ However, there is a wide range of predisposing conditions that have been delineated, including prolonged immobilization, trauma, obesity, hypercoagulable states, use of oral contraceptives or hormonal therapy, prior history of SVT or DVT, intravenous catheter use, malignancies, and autoimmune disorders. ${ }^{3,5,6}$ In particular, patients identified with Behcet's and Buerger's disease have been highlighted in recent reviews as being particularly susceptible to SVT. ${ }^{7}$

SVT is characterized by the combination of thrombosis and inflammation in a superficial vein, and involves the great saphenous vein in up to $60 \%-80 \%$ of cases. Cases involving the small saphenous vein are next in frequency, occurring in $10 \%-20 \%$ of cases, followed by upper extremity veins. Thrombosis normally occurs as a sequelae of "phlebitis" or inflammation (not infection) of the vein. However, secondary "phlebitis" is also seen. The pathophysiology of SVT can be classified in terms of external trauma, internal direct endothelial trauma, vein wall inflammation, and primary hematologic changes. External trauma can result from direct external force or compression, either from blunt traumatic injury or externally applied dressings. A superficial vein exposed to external force can sustain endothelial damage with resulting edema and leukocyte activation that predisposes to thrombosis. ${ }^{8}$ Prominent varicose veins are both more likely to have decreased flow rates and venous stasis, as well as local external injury, contributing to the higher incidence of SVT.

Internal trauma involves a direct endothelial injury leading to activation of the same inflammatory response seen in external trauma, with similar outcomes. The inciting event is often related to routine intravenous procedures, including phlebotomy and intravenous infusions. The length of time a catheter is in place is related to the rate of SVT. In addition, infusion of hypertonic solutions can directly injure the endothelium. Commonly implicated drugs are diazepam and pentobarbitone, both of which can cause a chemical inflammation. Infusions in areas of slower venous return, such as in more distal veins, are also more likely to result in SVT. The patient most commonly presents with increasing pain and tenderness directly at the catheter site and erythema. The intravenous catheter can also serve as the nidus for suppurative superficial venous thrombosis. Thrombus which forms around a catheter tip thus becomes secondarily infected and can lead to sepsis.

Suppurative superficial venous thrombosis is characterized by pus at the injection site, a tender, erythematous extremity, and possibly systemic signs, including fever, leukocytosis, and hemodynamic compromise. Commonly cultured organisms include Staphylococcus aureus, Pseudomonas, Klebsiella, Enterococcus, Fusibacterium, and Candida. ${ }^{7}$ Treatment requires prompt removal of the offending catheter, with drainage of any concomitant abscess and initiation of the appropriate intravenous antibiotics. Excision of the vein is not routinely necessary to treat the infection.

Vein wall inflammation can be a primary process, such as is seen in Buerger's disease, or secondary to adjacent inflammatory changes. Buerger's disease characteristically involves the small arteries and veins of the extremities, and biopsy findings of acute SVT involving all three layers of the vessel wall can confirm the diagnosis. Adjacent inflammation with resultant SVT can be due to trauma, infection, with the previously discussed septic thrombosis, or adjacent malignant disease. Because some tumors grow along the line of draining veins, this can result in SVT, and, in fact, malignancy is reported in up to $13 \%-18 \%$ of patients with SVT. ${ }^{89}$ Moreover, while the overall incidence of SVT in nonvaricose veins is much lower, the presence of SVT in nonvaricose veins may be associated with a risk of malignancy and, as such, merits additional evaluation as clinically indicated. ${ }^{10}$ Mondor's disease is a specific entity that describes SVT of the thoracoepigastric vein of the breast and chest wall, most commonly associated with breast cancers or a hypercoagulable state. Nonsteroidal anti-inflammatory drugs and warm compresses are the recommended treatment in these cases, although the underlying process must also be evaluated. In males, Mondor's disease has been used to describe SVT of the dorsal vein of the penis. If symptoms fail to improve with nonsteroidal anti-inflammatory drugs, resection of the dorsal penile vein is occasionally indicated.

Migratory SVT is characterized by repeated thromboses of superficial veins at varying sites. Described by Trousseau, when associated with cancer, migratory thrombophlebitis can occur years before a cancer diagnosis is made. Although it can also be seen with some of the vasculitides, a diagnosis of migratory SVT merits further investigation for an occult malignancy. ${ }^{8}$

Hypercoagulability may be associated with SVT in as many as $35 \%$ of patients. ${ }^{11}$ Hypercoagulable disorders associated with SVT include factor $\mathrm{V}$ Leiden mutation (the most common), 20210 A gene mutation, abnormal plasminogen, tissue plasminogen activator disorders, lupus anticoagulant, and anticardiolipin antibody syndrome. In addition, primary blood diseases, including polycythemia, thrombocythemia, and sickle cell disease, also have been implicated as strong risk factors for the development of SVT. 
We have previously reported that patients with a hypercoagulable disorder were significantly more likely to develop later acute DVT $(P<0.02)$, but that recurrence of SVT was not more likely. Patients with SVT should be subjected to a hypercoagulable workup using the same criteria as with acute DVT. In addition, screening for underlying diseases, such as malignancy or vasculitis, with mammography, colonoscopy, and appropriate radiologic studies is performed as needed.

The prevalence of associated acute DVT in patients presenting with SVT is estimated to $6.8 \%-40 \% .^{12-14}$ The reason for the range of associated acute DVT is because of the wide variation in study design, patient characteristics, symptomatic status, type of SVT, inpatient versus outpatient setting, indications, and whether or not any noninvasive testing was performed. A recent study of 788 patients with SVT links several factors as predictors for concurrent DVT. This includes, as expected, active cancer, as well as inpatient status, age greater than 75 years, and SVT of nonvaricose veins. ${ }^{15}$ In our previous experience with outpatients diagnosed with SVT, the incidence of acute DVT was $13 \%{ }^{16}$ However, the incidence varied from $6.3 \%$ in patients with varicose veins, $33 \%$ in patients without varicose veins, and $40 \%$ in patients with a previous history of DVT. The occurrence of concomitant pulmonary embolism is also variable, from $0.5 \%$ to $4 \%$ in symptomatic patients, increasing to $33 \%$ when a lung scan is performed. ${ }^{17,18}$

\section{Diagnosis}

Patients typically present with tender erythematous areas overlying a superficial vein. This may be warm to touch, with a palpable mass and surrounding edema. The vein may be visibly distended proximal to the thrombosis. Patients may exhibit signs of chronic venous disease, with visible varicosities, skin pigmentation, or palpable cords.

Pain can develop and progress quickly over several hours, and can be severe. The entire length of the great saphenous vein can be affected, or isolated segments can be involved. Isolated segments can be seen when associated with indwelling catheters. Direct trauma to the area is often elicited in the patient history, and can take the form of actual external trauma to the area or simply instrumentation with catheter placement and/or drug administration.

It is prudent to perform a duplex ultrasound scan in patients suspected of having SVT. Patients with catheterassociated peripheral SVT of the upper limbs or minor SVT associated with direct trauma may not require a duplex ultrasound scan. The extent of superficial thrombosis should be documented, and evaluation for a concomitant DVT must be completed. Some patients warrant a hypercoagulable or malignancy evaluation particularly when SVT is not associated with instrumentation or varicosities. The clinical history, risk factors, and family history guide the extent of this evaluation, which may include simply screening for inherited thrombophilias or more extensive malignancy, or vasculitis screening.

\section{Treatment algorithm}

The vast majority of patients with SVT are treated symptomatically with local heat, anti-inflammatory agents, and compression. Treatment of SVT is aimed at decreasing pain, decreasing inflammation, and preventing complications and recurrence. In cases secondary to an intravenous catheter or device, the offending foreign body must be removed. However, the treatment depends on the location, presence of concomitant acute DVT, first episode versus recurrence, presence or absence of varicose veins, and history of hypercoagulable disorders (Figure 1). As mentioned, the incidence of acute DVT is reported to be as high as $40 \%$. Therefore, other than in patients with SVT associated with a local varix or an intravenous catheter or device, obtaining a duplex ultrasound scan of the extremity is helpful early in the course of treatment in most patients. Duplex ultrasound findings in acute DVT consist of noncompressibility of the vein, partial or absent color flow in the lumen, visualization of luminal thrombus, absence of phasic variation with respiration and lack of augmentation of venous flow with calf compression and usually dilatation of the vein. In addition, special attention should be paid to the status of the SFJ both in terms of its relative distance from the thrombosed segment and whether the junction is incompetent. Patients with SVT in close proximity to the SFJ or saphenopopliteal junction are generally anticoagulated, even though the evidence for progression into the deep venous system is weak. Patients with SVT and varicose veins and reflux demonstrated by duplex ultrasound scan may be initially treated nonoperatively, although a large number will require surgery. Indeed, some authors strongly advocate considering surgery first in cases of SVT involving the axial veins with documented reflux of the SFJ. In order to minimize morbidity and loss of work, it may be more expeditious to remove the affected saphenous vein along with the varicose veins. In patients without varicose veins, the probability of an underlying thrombophilic disorder is high, and investigation is necessary prior to the use of anticoagulants. 


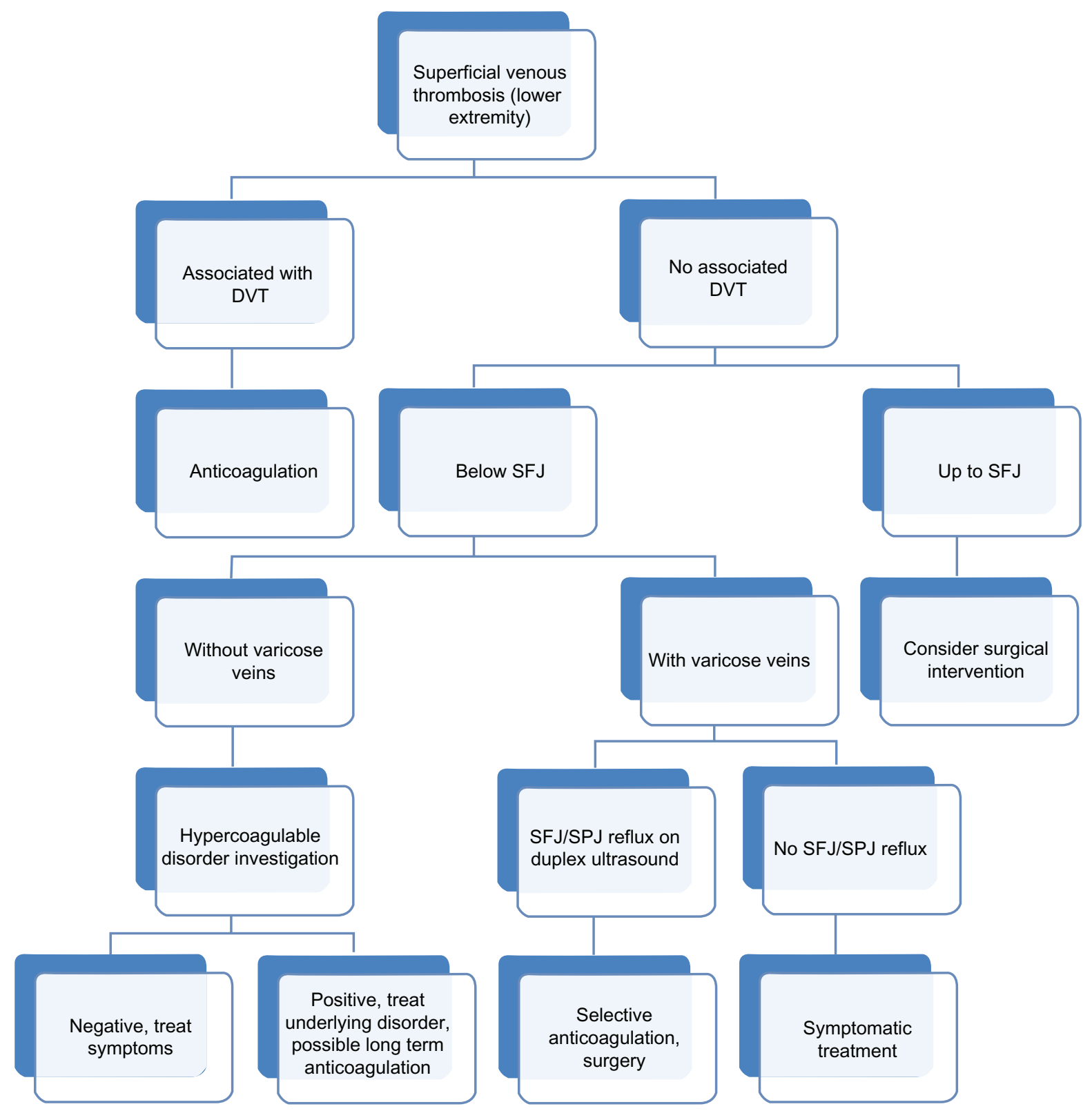

Figure I Management plan for superficial venous thrombosis of the lower extremity.

Abbreviations: DVT, deep venous thrombosis; SFJ, saphenofemoral junction; SPJ, saphenopopliteal junction.

\section{Newer and evolving treatment approaches}

The traditional approach for the vast majority of patients has focused on alleviating symptoms with warm compresses, nonsteroidal anti-inflammatory drugs, and compression garments when necessary. Frequent ambulation rather than bed rest is also advised. A change in strategy in managing certain patients with specific problems associated with SVT has materialized in recent reports. A personalized, individualized treatment plan seems to be the best approach towards this group of patients because the variation in presentation, risk factors, and extent of involvement is considerable.

\section{SVT of the great saphenous vein}

We have reported that almost $82 \%$ of outpatients with SVT were found to have involvement of the superficial axial veins. ${ }^{16}$ SVT in the superficial axial veins (great saphenous vein or small saphenous vein) is generally considered to warrant aggressive treatment with low molecular weight heparin to prevent extension into the deep venous system, particularly if the SVT is close to the junction with the common femoral or popliteal veins. Chengelis et al reported a $24 \%$ incidence of progression to DVT in 263 patients with proximal great saphenous vein involvement or SFJ involvement (14\%) who were not treated with anticoagulants. ${ }^{19}$ The majority (85.7\%) of DVT in this subgroup 
was observed to develop by extension through the SFJ, and the remaining extended via thigh perforating veins. The group with distal saphenous vein or variceal involvement only had a $4 \%$ incidence of DVT prompting the authors to recommend anticoagulants for all patients with proximal great saphenous vein or SFJ thrombus. The POST Study Group evaluated 844 patients with SVT, 25\% of whom had concomitant DVT. Of the 600 patients who initially presented without DVT or pulmonary embolism, $10.2 \%$ developed some thromboembolic complications within the three-month follow-up period. ${ }^{20}$

In another series of 20 selected patients with SVT of the great saphenous vein within $1 \mathrm{~cm}$ of the SFJ, there was a $40 \%$ incidence of concurrent acute DVT. ${ }^{13}$ Lohr et al reported on 43 patients who had SVT of the great saphenous vein within $3 \mathrm{~cm}$ of the SFJ by duplex ultrasound scan. ${ }^{21}$ Prandoni et al randomized patients with thrombus extending to within $3 \mathrm{~cm}$ of the SFJ to either therapeutic $(\mathrm{n}=83)$ or prophylactic $(n=81)$ doses of subcutaneous nadroparin for 30 days, with extension defined as progression of the SVT by at least $2 \mathrm{~cm}$ and closer than $3 \mathrm{~cm}$ from the SFJ.22 After treatment, two DVT events occurred in the prophylactic group and three in the therapeutic group, with only one symptomatic pulmonary embolism in the therapeutic group. Overall, $8.6 \%$ of patients in the prophylactic group and $7.2 \%$ in the treatment group developed either SVT progression or venous thromboembolic complications during the three-month follow-up period.

A meta-analysis of largely retrospective studies by Sullivan et al suggests that anticoagulation for the treatment of above-knee great saphenous vein involvement appears to be a reasonable option. ${ }^{23}$ A recent randomized, doubleblind trial in over 3000 patients demonstrated a reduction of both pulmonary embolism and DVT from $1.3 \%$ to $0.2 \%(P<0.001)$ for patients with SVT treated with fondaparinux versus placebo once a day for 45 days. Bleeding complications were similar in both groups. ${ }^{24}$ Indeed, Kitchens emphasizes that treatment of SVT is equivalent to that for venous thromboembolism with recommendations of full anticoagulation and further evaluation and imaging only if treatment would be altered, using the same considerations in evaluating SVT as are routinely used for DVT. ${ }^{25}$ Preliminary results suggest a lower recurrence rate, but whether anticoagulation enhances patency of the great saphenous vein allowing for later use as a conduit remains unresolved.

\section{Recurrence of SVT}

Recurrence of SVT is reported in $15 \%-20 \%$ of patients. ${ }^{26}$ Hafner et $\mathrm{al}^{27}$ in a series of 324 patients reported a prior history of similar episodes in $15 \%$ of patients. Ascer et $\mathrm{al}^{28}$ described a previous history of SVT in $20 \%$ in their series of 20 patients. The Austrian Study on Recurrent Venous Thromboembolism reported a 30-month follow-up of 615 patients with venous thromboembolism treated for three months with anticoagulants to look at the incidence and various factors that led to SVT. ${ }^{29}$ The overall incidence of recurrence in this group was $7.5 \%$. Patients who developed SVT were older, were followed up for longer, had a higher body mass index, and had a higher level of factor VIII (but not factor V Leiden). The recurrence rate obviously varies with the risk factors in the study cohort. Our previous report identified a $6 \%$ recurrence rate in 60 outpatients with SVT, and these episodes occurred at a mean of 57.8 days (standard deviation 50.09, median 39 days). ${ }^{15}$ Recurrent SVT was much more likely in patients with thrombosis of the tributaries as compared with patients without thrombosis of tributaries $(P<0.0008)$.

\section{Randomized studies with low molecular weight heparin}

There are multiple reasons for considering anticoagulants as a treatment option in patients with acute SVT. The most common indication is the presence of or increased risk of associated acute DVT/pulmonary embolism due to the location of SVT. In such cases, prophylaxis for about four weeks is often recommended. ${ }^{1}$ In a multicenter study of 117 patients randomized between fixed-dose low molecular weight heparin, dosemonitored low molecular weight heparin, and oral naproxen for six days, local heat and redness were less in both the low molecular weight heparin groups compared with naproxen. In addition, both low molecular weight heparin groups had less persistence of signs and symptoms at eight weeks. ${ }^{30}$ In another large randomized study, 427 patients with acute SVT of the legs were randomized to a fixed dose of enoxaparin $40 \mathrm{mg}$, enoxaparin $1.5 \mathrm{mg} / \mathrm{kg}$ body weight, an oral nonsteroidal antiinflammatory drug, ie, tenoxicam $20 \mathrm{mg}$, or placebo once daily for 8-12 days. ${ }^{31}$ Compared with placebo, the active treatment groups showed a much lower incidence of acute DVT and SVT by day 12 (placebo $30.6 \%$ versus $8.3 \%$ for fixed-dose enoxaparin, $6.9 \%$ for weight-based enoxaparin, and $14.9 \%$ for tenoxicam). At three months, the active treatment groups still retained an advantage versus placebo for combined DVT and SVT. Overall, there is evidence indicating that both low molecular weight heparin and nonsteroidal anti-inflammatory drugs reduce the progression of SVT or recurrence.

\section{Current role of surgery}

One of the earliest and largest experiences with SVT treated surgically was of 163 patients at the Mayo Clinic, 
with a recurrence rate of only $4.3 \%$ and a mean follow-up of five years. Interestingly, two thirds of these patients received postoperative anticoagulants. ${ }^{33}$ The safety of surgical intervention was also established by Husni and Williams, who reported 135 patients with SVT treated surgically with no postoperative pulmonary embolism. ${ }^{26}$ Sullivan et al reviewed several series of patients with SVT of the above-knee great saphenous vein and compared the outcome of treatment with anticoagulation, ligation of the SFJ, and ligation and stripping of the great saphenous vein. ${ }^{23}$ They concluded that ligation and stripping of the great saphenous vein was superior to ligation alone or anticoagulation in terms of rapid symptom relief. Anticoagulation was noted to be somewhat superior for minimizing complications and preventing subsequent DVT and pulmonary embolism.

A randomized trial with 70 patients in each of six groups showed that complete vein stripping or treatment with unfractionated heparin, low molecular weight heparin, or warfarin were superior to compression alone or in addition to flush ligation of the saphenous vein for the end point of SVT extension at three months. ${ }^{34}$ Another trial compared enoxaparin $1 \mathrm{mg} / \mathrm{kg}$ twice daily for one week, and then daily for three weeks with saphenofemoral ligation performed under local anesthesia with a follow-up of six months. ${ }^{35}$ Pulmonary embolism occurred in two patients $(6.7 \%)$ in the surgery group versus no venous thromboembolism in the enoxaparin group. SVT occurred in $10 \%$ of the enoxaparin group and in one patient $(3.3 \%)$ in the surgery group. Similar rates of SVT progression but higher rates of venous thromboembolism and complications were observed with surgical therapy compared with anticoagulation for SVT. ${ }^{23}$

The use of simple ligation or disconnection of the SFJ or saphenopopliteal junction for thrombus close to the SFJ/ saphenopopliteal junction, contraindication to anticoagulants, or progression as an acute measure despite anticoagulant therapy is no longer relevant for patients who need surgical intervention. In most cases, ligation and concomitant excision of the affected vein with the thrombosed vein branches, if feasible, can be safely performed. The complete operation can remove existing varicosities, provide cosmetic relief, relieve pain, prevent recurrences, and shorten the recovery time associated with periods of anticoagulation, with minimal morbidity. ${ }^{23}$

\section{Conclusion}

The most recent American College of Chest Physicians guidelines state "For patients with spontaneous superficial vein thrombosis, we suggest prophylactic or intermediate doses of low molecular weight heparin (Grade 2B) or intermediate doses of UFH (Grade 2B) for at least 4 weeks." ${ }^{32}$ The decision to use anticoagulant treatment for patients with SVT is not controversial in those patients with known thrombophilic disorders, continued symptoms, progression, and recurrent episodes. Patients without clinical risk factors such as immobilization, obesity, malignancy, or hormonal therapy, or immobilization and associated SVT certainly are at lower risk to develop complications of venous thromboembolism and therefore a less aggressive stance may be justified. If the axial great saphenous vein or small saphenous vein system is involved but the thrombus is not in proximity to the SFJ or saphenopopliteal junction, standard measures including heat, anti-inflammatory drugs, and ambulation are advised. For SVT at or close to the SFJ, the general recommendation (without solid evidence) is low molecular weight heparin. A repeat duplex ultrasound scan may be advisable in almost all circumstances if the symptoms persist or worsen. The role of surgical excision, or exclusion of the vein, becomes important when dealing with refractory or recurrent cases of SVT. However, surgery does not address any concomitant DVT, a phenomenon which has been increasingly appreciated in recent literature. This review of the literature emphasizes the wide variation in presentation, risk factors, associated DVT, or pulmonary embolism, and extent of local involvement of the superficial axial veins. It becomes clear that a more personalized, individualized approach to the patient with SVT is necessary.

\section{Future directions}

Because the reported incidence of acute DVT in patients with SVT can be as high as $40 \%$, a multicenter, prospective, randomized study is needed to clarify the role of anticoagulant therapy as well as the optimal dosing and duration of treatment. In patients with recurrent SVT, such as the patient population with thrombophilic disorders, the use of the newer oral anticoagulants will need to be clarified. Even though there are some studies comparing nonoperative treatment and surgical intervention, we need a large multicenter study to look at the recurrence rate, morbidity, and cost-benefit analysis to elucidate the exact role of surgical intervention. If surgical therapy is beneficial, which specific groups should be considered? These unresolved issues are prime targets for future research to allow for safer and more cost-efficient management of patients with SVT.

\section{Disclosure}

The authors report no conflicts of interest in this work. 


\section{References}

1. Di Nisio M, Wichers IM, Middeldorp S. Treatment for superficial thrombophlebitis of the leg. Cochrane Database Syst Rev. 2007;2: CD004982.

2. De Wees M. Non-operative treatment of acute superficial thrombophlebitis and deep femoral thrombosis. In: Ernst CB, Stanley JC, editors. Current Therapy in Vascular Surgery. Philadelphia, PA: BC Decker; 1991.

3. Coon WW, Willis PW 3rd, Keller JB. Venous thromboembolism and other venous disease in the Tecumseh community health study. Circulation. 1973;48:839-846.

4. Marchiori A, Mosena L, Prandoni P. Superficial vein thrombosis: Risk factors, diagnosis, and treatment. Semin Thromb Hemost. 2006;32: 737-743.

5. Leon L, Giannoukas AD, Dodd D, et al. Clinical significance of superficial vein thrombosis. Eur J Vasc Endovasc Surg. 2005;29:10-17.

6. Meissner MH, Wakefield TW, Ascher E, et al. Acute venous disease: Venous thrombosis and venous trauma. J Vasc Surg. 2007;46 Suppl: 25S-53S.

7. Hingorani AP, Ascher E. Superficial thrombophlebitis. In: Cronenwett J, Johnston K, editors. Rutherford's Vascular Surgery. Philadelphia, PA: Elsevier; 2010.

8. Browse NL, Burnand KG, Thomas ML. Diseases of the Veins: Pathology, Diagnosis and Treatment. London, UK: Hodder and Stoughton; 1988.

9. Krause U, Kock HJ, Kroger K, et al. Prevention of deep venous thrombosis associated with superficial thrombophlebitis of the leg by early saphenous vein ligation. Vasa. 1998;27:34-38.

10. Gillet JL, Allaert FA, Perrin M. Superficial thrombophlebitis in non varicose veins of the lower limbs. A prospective analysis in 42 patients. J Mal Vasc. 2004;29:263-272. French.

11. Hanson JN, Ascher E, DePippo P, et al. Saphenous vein thrombophlebitis (SVT): A deceptively benign disease. J Vasc Surg. 1998;27:677-680.

12. Belcaro G, Nicolaides AN, Errichi BM, et al. Superficial thrombophlebitis of the legs: A randomized, controlled, follow-up study. Angiology. 1999;50:523-529.

13. Ascher E, Lorensen E, Pollina RM, Gennaro M. Preliminary results of a nonoperative approach to saphenofemoral junction thrombophlebitis. J Vasc Surg. 1995;22:616-621.

14. Skillman JJ, Kent KC, Porter DH, Kim D. Simultaneous occurrence of superficial and deep thrombophlebitis in the lower extremity. J Vasc Surg. 1990;11:818-824.

15. Galanaud JP, Genty C, Sevestre MA, et al; for OPTIMEV SFMV investigators. Predictive factors for concurrent deep-vein thrombosis and symptomatic venous thromboembolic recurrence in case of superficial venous thrombosis. The OPTIMEV study. Thromb Haemost. 2011;105:31-39.

16. Gorty S, Patton-Adkins J, Dalanno M, Starr JE, Dean S, Satiani B. Superficial venous thrombosis of the lower extremities: Analysis of risk factors, and recurrence and role of anticoagulation. Vasc Med. 2004;9:1-6.

17. Decousus H, Epinat M, Guillot K, et al. Superficial vein thrombosis risk factors, diagnosis and treatment. Curr Opin Pulm Med. 2003;9: 393-397.

18. Verlato F, Zucchetta P, Prandoni P, et al. Unexpected high rate incidence of pulmonary embolism in patients with superficial thrombophlebitis of the thigh. J Vasc Surg. 1999;30:1113-1115.

Vascular Health and Risk Management

\section{Publish your work in this journal}

Vascular Health and Risk Management is an international, peerreviewed journal of therapeutics and risk management, focusing on concise rapid reporting of clinical studies on the processes involved in the maintenance of vascular health; the monitoring, prevention and treatment of vascular disease and its sequelae; and the involvement of
19. Chengelis DL, Bendick PJ, Glover JL, Brown OW, Ranval TJ. Progression of superficial venous thrombosis to deep venous thrombosis. J Vasc Surg. 1996;24:745-749.

20. Decousus H, Quéré I, Presles E, et al. for the POST Study Group. Superficial thrombophlebitis and venous thromboembolism: A large prospective epidemiological study. Ann Intern Med. 2010;152:218-224.

21. Lohr JM, McDevitt DT, Lutter KS, Roedersheimer LR, Sampson MG. Operative management of greater saphenous thrombophlebitis involving the saphenofemoral junction. Am J Surg. 1992;164:269-274.

22. Prandoni P, Tormene D, Pesavento R. High vs low doses of lowmolecular-weight heparin for the treatment of superficial vein thrombosis of the legs: a double-blind, randomized trial. J Thromb Haemost. 2005;3:1152-1157.

23. Sullivan V, Denk PM, Sonnad SS, Eagleton MJ, Wakefield TW. Ligation versus anticoagulation: Treatment of above-knee superficial thrombophlebitis not involving the deep venous system. J Am Coll Surg. 2001;193:556-562.

24. Decousus H, Prandoni P, Mismetti P, et al; for the Calista Study Group. Fondaparinux in the treatment of superficial-vein thrombosis of the leg. N Engl J Med. 2010;363:1222-1232.

25. Kitchens CS. How to treat superficial venous thrombosis. Blood. 2011; 117:39-44.

26. Husni EA, Williams WA. Superficial thrombophlebitis of lower limbs. Surgery. 1982;91:70-74.

27. Hafner CD, Cranley JJ, Krause RJ, Strasser ES. A method of managing superficial thrombophlebitis. Surgery. 1964;55:201-206.

28. Ascer E, Lorensen E, Pollina RM, Gennaro M. Preliminary results of a nonoperative approach to saphenofemoral junction thrombophlebitis. J Vasc Surg. 1995;22:616-621.

29. Schonauer V, Kyrle PA, Weltermann A, et al. Superficial thrombophlebitis and risk for recurrent venous thromboembolism. J Vasc Surg. 2003; 37:834-838.

30. Titon JP, Auger D, Grange P, et al. Therapeutic management of superficial venous thrombosis with calcium nadroparin. Dosage testing and comparison with a non-steroidal anti-inflammatory agent. Ann Cardiol Angeiol (Paris). 1994;43:160-166. French.

31. The Superficial Thrombophlebitis Treatment by Enoxaparin Study Group. A pilot randomized double-blind comparison of a lowmolecular-weight heparin, a non-steroidal anti-inflammatory agent, and placebo in the treatment of superficial vein thrombosis. Arch Intern Med. 2003;163:1657-1663.

32. Kearon C, Kahn SR, Agnelli G, Goldhaber SZ, Raskob G, Comerota AJ. Antithrombotic therapy for venous thromboembolic disease: ACCP evidence-based clinical practice guidelines. Chest. 2008;133(6 Suppl):454S-455S.

33. Lofgren EP, Lofgren KA. The surgical treatment of superficial thrombophlebitis. Surgery. 1981;90:49-54.

34. Belcaro G, Nicolaides AN, Errichi BM, et al. Superficial thrombophlebitis of the legs: A randomized, controlled, follow-up study. Angiology. 1999;50:523-529.

35. Lozano FS, Almazan A. A Low-molecular-weight heparin versus saphenofemoral disconnection for the treatment of above-knee greater saphenous thrombophlebitis: A prospective study. Vasc Endovascular Surg. 2003;37:415-420.

metabolic disorders, particularly diabetes. This journal is indexed on PubMed Central and MedLine. The manuscript management system is completely online and includes a very quick and fair peer-review system, which is all easy to use. Visit http://www.dovepress.com/ testimonials.php to read real quotes from published authors. 